\title{
Development of Tubular Cardiovascular Phantom System for Pulse Transit Time Simulation
}

\author{
Wan Suhaimizan Wan Zaki, Ricardo Correia, Serhiy Korposh, Barrie R Hayes-Gill, Stephen P \\ Morgan
}

\begin{abstract}
This paper presents on the development of a tubular cardiovascular phantom system to simulate pulse transit time (PTT). The PTT defined as the delay time between two pulses in one cardiac cycle has been shown to be promising method for cuffless continuous blood pressure (BP) measurement. However most of the PTT measurement was performed on human subjects, thus giving a difficulty in validating sensor performance due to variability of BP. Therefore, a cardiovascular phantom system was proposed for simulate the PTT measurement. An electronic controlled module was developed to control pump operation for pulse generation. Plastic optical fibre (POF) sensors were used to measure the pulse signal on the flexible tube and the results were compared with an in-line pressure sensor. In this experiment, the delay time between two pulses were calculated offline using Matlab software and correlated with pulse pressure. The result demonstrate that the pulse delay time recorded by both sensors decreased with increase of pulse rate and pulse pressure. These results on the phantom study showed similar pattern to the human model, thus indicating that the system is able to simulate PTT for sensor validation purposes.
\end{abstract}

Index Terms: Fibre optics, Phantom, Pulse Transit Time.

\section{INTRODUCTION}

In general, a laboratory model or a phantom is developed for a calibration and validation of new device. A phantom system is able to enhance understanding of the basic principles of a physiological system such as pulse wave propagation in a blood vessel [1] and PPG characteristics on different tissues properties [2]. A physical model of cardiovascular system has been widely developed mainly focused on a photoplethysmography (PPG) pulse waveform [3],[4] and to simulate pulse wave velocity (PWV) measurement [5],[6] leading to a pulse transit time (PTT) measurement system. A pulsatile waveform was generated either by a commercial pulsatile pump [7],[8] or custom motorised pump [9].

Revised Manuscript Received on June 22, 2019.

Wan Suhaimizan Wan Zaki, Optics and Photonics Group, Faculty of Engineering, University of Nottingham, NG7 2RD, United Kingdom, Department of Electronic Engineering, Universiti Tun Hussein Onn Malaysia, 86400 Johor, Malaysia.

Ricardo Correia, Optics and Photonics Group, Faculty of Engineering, University of Nottingham, NG7 2RD, United Kingdom.

Serhiy Korposh, Optics and Photonics Group, Faculty of Engineering, University of Nottingham, NG7 2RD, United Kingdom.

Barrie R Hayes-Gill, Optics and Photonics Group, Faculty of Engineering, University of Nottingham, NG7 2RD, United Kingdom.

Stephen $\mathbf{P}$ Morgan, Optics and Photonics Group, Faculty of Engineering, University of Nottingham, NG7 2RD, United Kingdom.
A flexible tube was exploited by some workers for pulse detection using ultrasound [1],[6] or by optical methods [7],[10]. A characteristic of the flexible tube is that it expands with increasing pulse pressure making it suitable to be applied for heart beat simulation [11] and for photoplethysmography (PPG) measurement [12]. In a human, the delay time between two pulses known as pulse transit time (PTT) is highly correlated with systolic blood pressure (SBP) [13],[14]. In addition, a physiological parameter such as heart rate and arterial stiffness also affects the PTT measurement, thus making it difficult to validate sensor performance due to a lack of control of the system. Therefore, in this study, a PTT phantom system was developed to mimic the human blood circulatory system and to provide useful information on PTT measurement.

The system was designed based on Windkessel model in representing arterial compliance [4]. The amount of air and pressure inside a custom-made chamber was controlled to simulate the arterial compliance in a human system. A highly flexible tube was exploited to the mimic the human blood vessel. A plastic optical fibre (POF) system which consists of LED as a light source and phototransistor for the light detector was used for pulse measurements. The flexible tube expanded with the corresponding volume change, thus increasing the light absorption and reducing the amount of reflected light detected by the phototransistor [11]. The pulse waveform detected by the POF were compared with a pressure sensor for validation purposes. The details of the designed PTT tubular phantom system and the results of the findings from the experimental testing is presented in this paper and demonstrates trends consistent with a human model.

\section{EXPERIMENTAL SETUP}

Figure 1 illustrates a schematics of a designed PTT phantom system and Figure 2 shown a photograph of the developed system. The system consists of a diaphragm pump (RS-385), two in-line pressure sensors (PS1 and PS2, PandoTECH, OU-19406-27), two flexible tubes (FT1 and FT2, T4X0.5ST40, Polymax, UK), two reservoirs (R1 and $\mathrm{R} 2)$, a one-way valve and a rotary valve (RV, FCH-M-PPLC, Bio-Tech). In this system, two PTTs are determined based on the pulse delay time detected by the two in-line pressure sensors (PS) and the plastic optical fibres (POF) respectively.

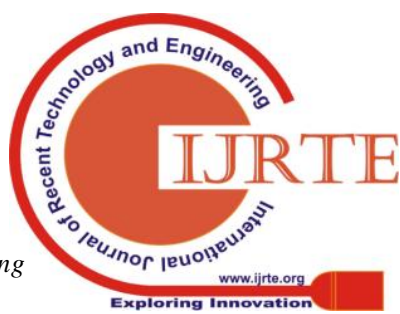


The first PTT is defined as the pulse delay time between PS1 and PS2 and the second PTT is defined as the pulse delay time between POF1 and POF2. The locations of the PS and POF are shown in Figure 1.

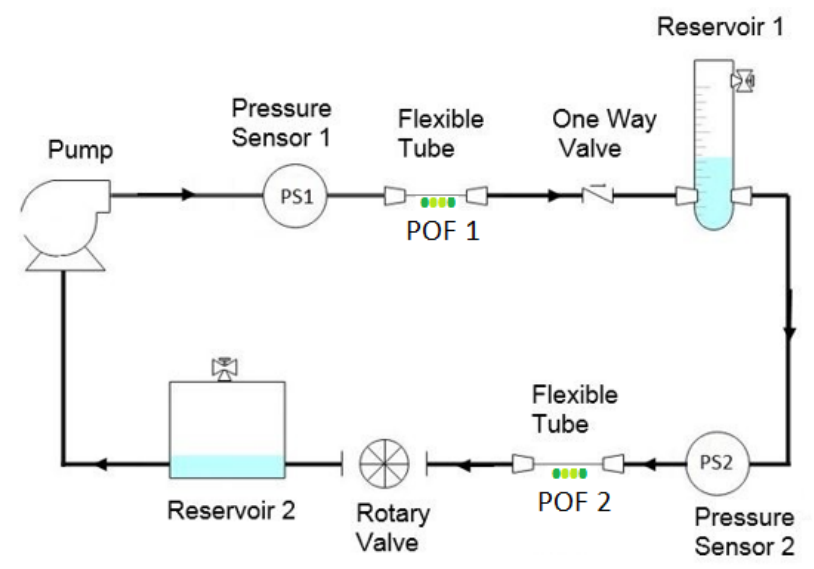

Fig. 1 Schematic of the designed PTT tubular phantom system.

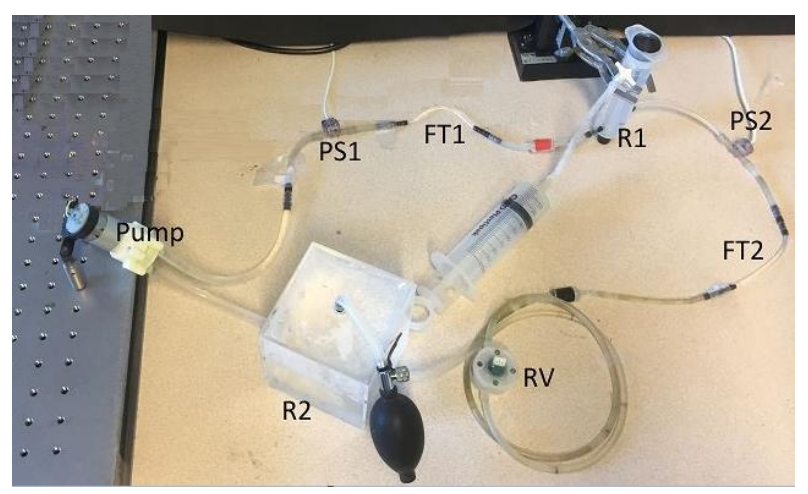

Fig. 2 Photograph of the PTT tubular phantom system.

PS1 and PS2 are the pressure sensor 1 and pressure sensor 2, FT1 and FT2 are the flexible tube 1 and flexible tube 2, R1 and R2 are reservoir 1 and reservoir 2 and $R V$ is a rotary valve.

The diaphragm pump is the heart of the system and works to circulate water around the phantom via a silicone tube (T4X1ST60, Polymax, UK). The total length of the silicone tube is $170 \mathrm{~cm}$. The distance between PS1 and PS2 was set to $45 \mathrm{~cm}$. The pulse wave was generated by switching the pump ON and OFF via the relay circuit (5V10A2 Channel Relay Module). A microcontroller (Arduino Mega 250) was programmed to trigger the relay module for pulse wave modulation.

The pulse pressure generated by the pump is detected by both of the pressure sensors (PS1 and PS2) and the plastic optical fibre (POF 1 and POF 2). The R1 was made from a modified syringe as shown in Figure 2. The function of R1 was to implement the Windkessel model in order to represent the arterial compliance [15] but also to buffer the pulse pressure from PS1 to PS2. According to the Windkessel analogy, the elastic arteries (aorta) can be represented by a chamber filled with water and a pocket of air. In this experiment, a chamber (R1) representing the vessel dimensions and the air within the chamber representing the vessel elasticity, or a 'windkessel' compliance. As it's pumped, the water compresses the air, which in turn pushes the water out of the chamber. A low arterial compliance was simulated by decreasing the amount of air and pressure inside the chamber. The pulse pressure is increased when compliance is reduced. Theoretically, the compliance is referred to the elasticity and extensibility of the structure and it is determined by dividing the change in volume with the change in pressure [16]. In the human body, the wall of the aorta expanded to accommodate the increase in blood volume if it is compliant. The compliance decreased with stiffening of the arterial wall, thus increasing the BP.

In addition, the pressure at the outlet of R1 increases with the increase of water level thus increasing the amplitude of pulse pressure. A 3-way stopcock (B. Braun Medical Inc.) was located at the top of the syringe (R1) and acts like a valve that connects ambient air to R1 (Figure 1). The water level increases as the stopcock opens due to the low pressure created inside R1. When the stopcock is in the closed position (closing the air path to R1) both the pressure and water level are maintained inside R1. A rotary valve (RV) was placed at the end of the PTT tubular phantom system to produce a high resistance point. Inside the RV, there is a nozzle with diameter of $2.5 \mathrm{~mm}$, which more than 2 times smaller than the RV port size. Therefore, the water that travels towards the nozzle become slow due to the sudden decrease in area. The water resistance increases with decrease in area, which affects the decay time of the pulse wave whilst mimicking a diastolic moment. According to the two-element Windkessel model, the exponential decay time depends on the product of peripheral resistance, $\mathrm{R}$, and total arterial compliance, $\mathrm{C}$ (the RC time) [16]. Based on this, the decay time will of course be longer with a high resistance. The last stage of the phantom system is reservoir 2 (R2). The R2 is made of a Perspex acrylic panel $(3 \mathrm{~mm}$ thick) of size $10 \mathrm{~cm}$ length $\times 10 \mathrm{~cm}$ width $\times 10 \mathrm{~cm}$ height. The pump pulls water from R2 which is discharged into the tubular phantom system. A sphygmomanometer bulb was connected to the top of R2 in order to pressurize the system. In this experiment, the phantom system was deployed as a PTT simulator and the performance of the POF sensors will be evaluated in this setting. The POF sensor was developed based on reflective mode PPG measurement. The POF probe consists of four fibres with two fibres for the transmitter and another two fibres for the receiver as shown in Figure 3. 


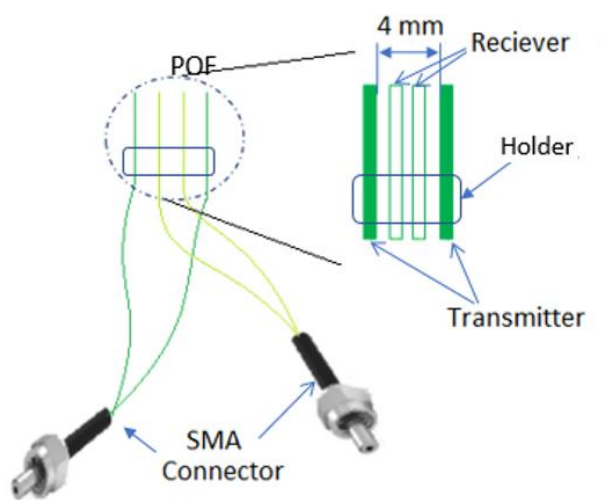

Fig. 3 Illustration of POF probe with SMA connector.

The fibre aligned in parallel with the distance between each fibre was set to $1 \mathrm{~mm}$. The POF were held together by a holder made from a wire jacket. The diameter of the POF was 500um and the external diameter of the wire jacket was $1 \mathrm{~mm}$. The SMA connectors were used to couple the transmitter fibres to the light source and to couple the receiver fibre to the photodetector.The tip of the fibres was cut to 45 degrees in order to illuminate the light and to receive the reflected light in a planar position. A highpowered green LED (Osram Opto Platinum Dragon) was used as a light source and phototransistor (TEMT-6000, Vishay) was used as a detector. The reflectance mode POF sensors was placed under the flexible tube (ID $=4 \mathrm{~mm}$, wall thickness $=0.4 \mathrm{~mm}$ ) for pulse wave measurement. Micropore tape was used to secure the flexible tube on top of the POF to prevent the structure from moving and creating motion artefacts. A black cloth was also deployed to cover the measurement area (the POF probe region) to prevent external ambient light sources from influencing the measurement. Two POF probes were used to measure the pulse wave with the distance between the two sensors was $45 \mathrm{~cm}$ to match the separation between PS1 and PS2.The green LED light from the transmitted fibre illuminated the tube and the reflected light from the upper wall of flexible tube was detected by the receiving fibre. The light intensity decreases as the tube expands due to the increase in the distance (and hence volume) between the receiver point and the reflection point. The receiver fibre sends the captured reflected light to aTEMT phototransistor light sensor module (photodetector) where the light intensity converted to a voltage for further processing. The voltage became less with less light intensity detected. The signals from the light sensor module were transferred to a computer via a NeXus data acquisition device (Nexus-10 Mark II). A block diagram for the PTT measurement is shown in Figure 4.
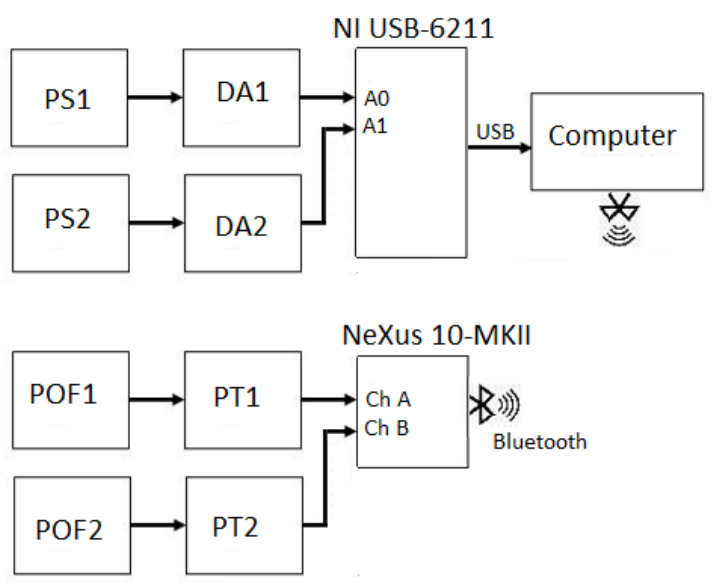

Fig. 4 Block diagram of pulse measurement on PTT phantom system. PS1 and PS2 are the pressure sensor 1 and pressure sensor 2, DA1 and DA2 are difference amplifier 1 and difference amplifier 2, A0 and A1 are analogue input 0and analogue input 1 for NI USB-62II data acquisition card, POF1 and POF2 are the plastic optical Fibre 1 and plastic optical Fibre 2, PT1 and PT2 are phototransistor 1 and phototransistor 2, Ch A and Ch $B$ are channel $A$ and channel $B$ of NeXus 10-MKII.

As shown in Figure 4, the output from the PS1 and PS2 were amplified using DA1 and DA2 respectively. The output from DA1 is connected to channel A0 and the output from DA2 is connected to channel A1 at the National Instruments (NI-USB 6211) Data Acquisition (DAQ) card. The NI USB 6211 has 8 analogue inputs (A0 to A7) with 16 bits ADC resolution. The signal then transferred to a computer via USB cable. Labview software was programmed to display and record the signals from the pressure sensors with a sampling frequency of $1000 \mathrm{~Hz}$. Simultaneously, the signal from the POF1 and POF2 were converted to the voltage via PT1 and PT2 respectively. Both signals were connected to channel A and Channel B of the NeXus 10-MKII device. The analogue signals then converted to digital signals through 24 bits ADC built inside the device and then sent it to the computer via Bluetooth. BioTrace+ software was deployed to display and record the signals measured by the POF probes with a sampling frequency of $256 \mathrm{~Hz}$. The stored data from the PS and POF probes were analyzed offline and manually aligned using Matlab for the pulse delay time measurement. The PTT measurement for the PS were calculated based on the pulse delay time between PS1 and PS2 and PTT for POF were calculated based on the pulse delay time between POF1 and POF2.

\section{RESULT}

The first experiment was the measurement of the pulse delay time with increase in water level inside reservoir R1. The water level varied from $25 \mathrm{ml}$ to $65 \mathrm{ml}$ by controlling the stopcock. 
The complete experiment was conducted with setting the pump's supplied voltage (Tenma, 72-10505) to 10 volt and the pulse rate was set to 60 pulses/minute. The pulse wave that travelled inside the tube was measured by the pressure sensors (PS1 and PS2) and POF probes (POF1 and POF2). Figure 5 shows the pulse waveform measured by PS and POF and Figure 6 shows the measurement of PTT with the pulse pressure.
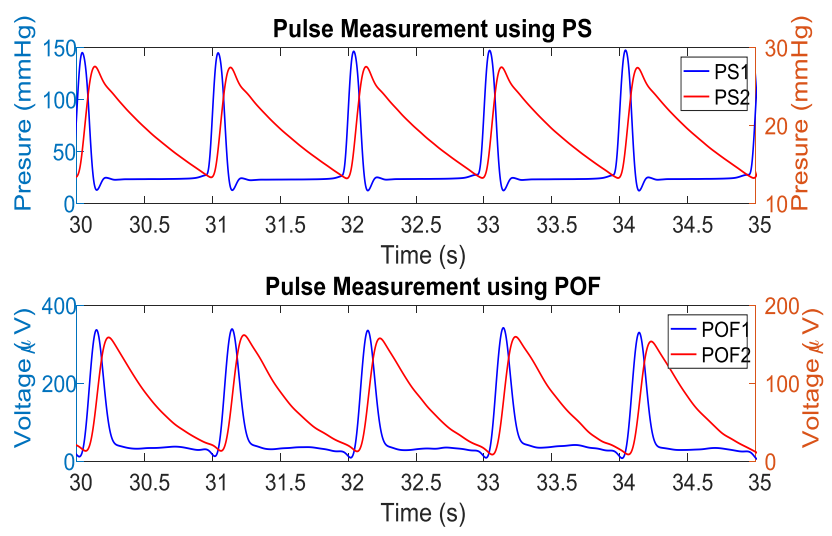

Fig. 5 Typical pressure sensor signals (PS1and PS2 top trace) and resulting optical plastic optical fibre sensors (POF1 and POF2 lower trace) when the pump power supply was set at $6 \mathrm{~V}$, pulse rate set at 60 pulse/min and water level R1 set to $25 \mathrm{ml}$

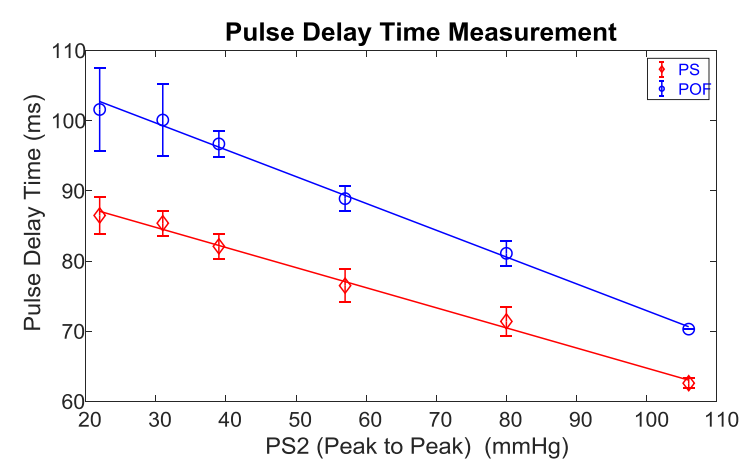

Fig. 6 Pulse delay time measurement on PS and POF with increased peak to peak pulse pressure at PS2 when the power supply was set at $10 \mathrm{~V}$ and pulse rate set at 60 pulse/min.

As shown in Figure 5 (upper trace), the $\mathrm{Y}$-axis represents water pressure detected by the pressure sensor (PS) measured in $\mathrm{mmHg}$. The expansion of the flexible tube due to the water flow was detected by the plastic optical fibre (POF) and is measured in microvolts. The output signals from the photodetector were inverted by the Matlab software for peak detection purpose. The PTT measurements were calculated based on an average of 15 seconds of pulses at each set of water levels. The results demonstrate that the PTT recorded by both sensors decreases with increase of pulse pressure. The sensitivity response for both PS and POF were calculated based on the slope of the graph and the result showed that sensitivity was $-0.2923 \mathrm{~ms} / \mathrm{mmHg}$ and $-0.3902 \mathrm{~ms} / \mathrm{mmHg}$ respectively. The results indicate that the POF was more sensitive in PTT measurement and gave a larger response compared to the PS.

In the literature, a pulse wave velocity (PWV) was reported to increase with the peak pressure [5]. The PWV was strongly affected by the tube elasticity and the velocity was higher on the stiff tube compared to the soft tubes [6]. In a typical blood circulatory system, the arterial compliance reduced due to a loss of vessel wall elasticity resulting in an increased in pulse wave velocity [17]. Aging is the main factor in human arterial compliance loss that increases the blood pressure [18]. It has been reported that the PTT was inversely related to the systolic blood pressure (SBP) i.e the PTT decreases with increase of SBP [19-20]. Overall, the PTT phantom is therefore able to simulate the arterial compliance condition and these results on the phantom study showed a similar pattern to the human model.

The next experiment conducted was to measure PTT with increase of pulse rate from 60 pulses/minute to 160 pulses/minute. This range of pulse rates covered the PTT variability over an acceptably wide range of heart rate as per Murakami et.al [21]. The pulse rate increased with the increase of triggered pulse frequency at the pump. The experiment was conducted with the pump supply voltage was set at $10 \mathrm{~V}$ and the initial water level in R1 was set at $25 \mathrm{ml}$. Figure 7 shows the pulse waveform measured by the $\mathrm{PS}$ and POF at 160 pulse/minute. The PTT responses to the pulse rate shown in Figure 8.
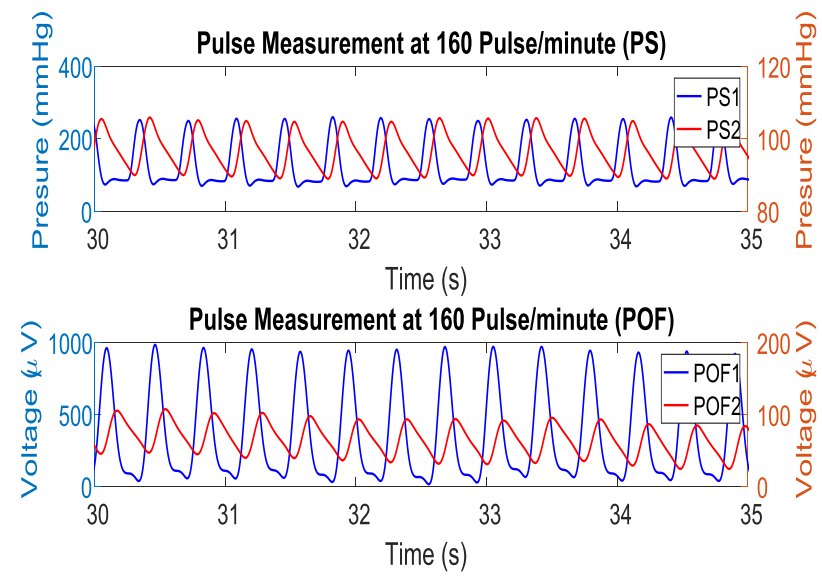

Fig. 7 Pulse waveform measured by both PS (upper trace) and POF (lower trace) when the pump power supply was set at $10 \mathrm{~V}$ and the pulse rate was set at 160 pulses/minute. 


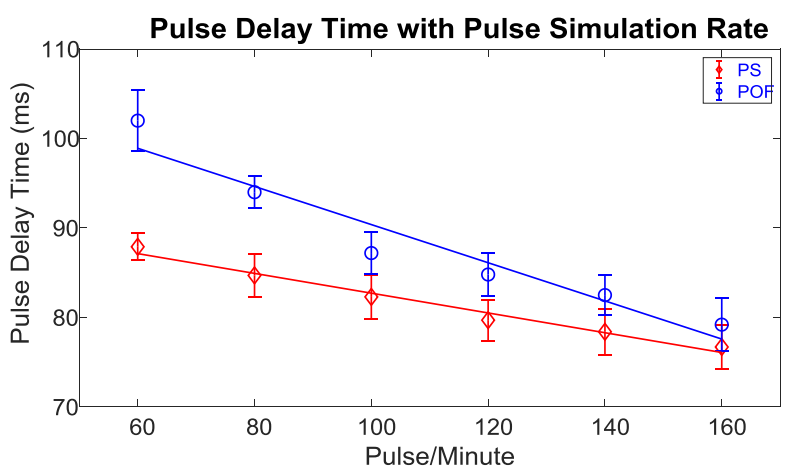

Fig. 8 The pulse delay time (PTT) measurement with increasing pulse rate with the pump power supply set at $10 \mathrm{~V}$.

The result in Figure 7 demonstrates that the PS and POF are able to respond to the pulse wave generated by the pump. The peak of the pulses was located and the delay time (PTT) between two pulses (PS1 to PS2 and POF1 to POF2) were calculated using Matlab software. The result in Figure 8 illustrates that PTT measured by both sensors (PS and POF) decreases with increases in pulse rate. Based on the graph slope, it was observed that the POFs have different sensitivity response especially at high pulse rate (above 100 pulse/minute). The flexible tube becomes stiffer at the higher pulse rate due to the increase in basal pressure that may result less sensitive in PTT measurement. It was observed that diastolic basal pressure measured at PS2 increased from $54 \mathrm{mmHg}$ to $85 \mathrm{mmHg}$ as the pulse rate increased from 100pulse/min to $160 \mathrm{pulses} / \mathrm{min}$. Further investigation showed that the pulse delay is not significantly influenced by the PS2 basal pressure, suggesting that the PTT decreased due to the increase in pulse rate. The sensitivity for PS measurement was almost constant over the entire range of pulse rate. The different mechanism detection may lead to different sensitivity response for both sensors. For example, the POF responded to mechanical change (tube expansion) in pulse detection while the PS responded to the water pressure inside the tube. Further, the tube material may affect the pulse delay response especially at higher pulse rate. The pressure sensor was placed inside Polysulfone material and connected to the silicone tube with wall thickness $1 \mathrm{~mm}$. Meanwhile, the POF sensor was placed at the flexible tube with wall thickness of $0.4 \mathrm{~mm}$. This condition showed that the tube connected to the PS was stiffer than the flexible tube at the POF. Therefore, the gradient for the POF showed less sensitivity at higher pulse rate, indicating that the tube becomes stiffer at the higher pulse rate due to the increase in diastolic basal pressure. The response however, from both sensors (PS and POF) were similar to the response observed in a human model. For example, in a human study, the PTT negatively correlated strongly with heart rate, i.e the PTT linearly decreases with an increase in heart rate [21]. As the heart rate increases, the cardiac output also increased, thus the BP increased linearly with the heart rate [22].

\section{CONCLUSION}

A tubular cardiovascular phantom system was successfully developed and tested on the POF sensors. Inline pressure sensors were used as a benchmark for the pulse delay time measurement. The performances of POF on pulse wave detection and PTT measurement were tested in two different condition. The first condition was by varying pulse amplitude by controlling the amount of water inside the reservoir $\mathrm{R} 1$. The pulse delay time between two pulses were calculated and a negative correlation with pulse pressure was observed. This finding agreed with the finding observed by other researchers in human studies, with PTT was reported inversely related to systolic blood pressure (SBP). The next configuration was to investigate the effect of pulse rate on pulse delay time. The pulse rate was controlled by microcontroller to trigger pulse frequency at the pump. The results showed that the pulse delay time (PTT) decreased with the increase in pulse rate. Similar responses have been observed in a human system with the PTT decreasing as the heart rate increased. Overall, the phantom system demonstrated excellent compliance with the human model and the performance of the POF sensors were successfully evaluated in this setting.

\section{ACKNOWLEDGMENT}

The corresponding author would like to acknowledge Ministry of Higher education Malaysia and Universiti Tun Hussein Onn Malaysia (UTHM) for the financial support during his study at the University of Nottingham, United Kingdom.

\section{REFERENCES}

1. Z. Wang, Y. Yang, L. J. Yuan, J. Liu, Y. Y. Duan, and T. S. Cao, "Noninvasive method for measuring local pulse wave velocity by dual pulse wave Doppler: In vitro and in vivo studies," PLoS One, vol. 10, no. 3, pp. 1-13, 2015.

2. T. J. Akl, M. A. Wilson, M. N. Ericson, and G. L. Coté, "Quantifying tissue mechanical properties using photoplethysmography," Biomed. Opt. Express, vol. 5, no. 7, pp. 2362, 2014

3. N. Stuban, M. Niwayama, and H. Santha, "Phantom with pulsatile arteries to investigate the influence of blood vessel depth on pulse oximeter signal strength," Sensors, vol. 12, no. 1, pp. 895-904, 2012.

4. D. Laqua, S. Pollnow, J. Fischer, S. Ley, and P. Husar, "A phantom with pulsating artificial vessels for non-invasive fetal pulse oximetry,'International Conference of the IEEEEngineering in Medicine and Biology Society (EMBC), 2014, pp. 5631-5634.

5. J. S. Lillie et al., "Pulse Wave Velocity Prediction and Compliance Assessment in Elastic Arterial Segments," Cardiovasc. Eng. Technol., vol. 6, no. 1, pp. 49-58, 2015.

I. Z. Apostolakis, P. Nauleau, C. Papadacci, M. D. McGarry, and E. E. Konofagou, "Feasibility and Validation of 4-D Pulse Wave Imaging in Phantoms and In Vivo," IEEE Trans. Ultrason. Ferroelectr. Freq. Control, vol. 64, no. 9, pp. 1305-1317, 2017.

6. H. Njoum et al., "Photoplethysmography for an independent measure of pulsatile pressure under controlled flow conditions," Physiol. Meas., vol. 38, no. 2, pp. 87-100, 2017.

7. K. Rhodea and G. Ennewa, "The measurement of blood flow from dynamic digital $\mathrm{x}$-ray images using a weighted optical flow algorithm: Validation in a moving-vessel flow phantom," Medical Image Understanding and Analysis, 2001,pp. 4-7. 
8. M.-H. Jun, Y. J. Jeon, J.-H. Cho, and Y.-M. Kim, "Pulse wave response characteristics for thickness and hardness of the cover layer in pulse sensors to measure radial artery pulse," Biomed. Eng. Online, vol. 17, no. 1, p. 118, 2018.

9. N. Stubán and M. Niwayama, “Adjustable fetal phantom for pulse oximetry,” Rev. Sci. Instrum., vol. 80, pp. 78-82, 2009.

10. D. Laqua, S. Ley, P. Husar, and I. Methods, "Development of a Phantom to Modulate the Maternal and Fetal Pulse Curve for Pulse Oximetry Measurements,"" Biomedical Engineering, vol. 57, pp. $803-$ 806, 2012.

11. J. P. Phillips, M. Hickey, and P. A. Kyriacou, "Evaluation of electrical and optical plethysmography sensors for noninvasive monitoring of hemoglobin concentration," Sensors, vol. 12, no. 2, pp. 1816-1826, 2012.

12. H. Jeong et al., "A Study on Estimat ing Blood Pressure during Body Postural Change based on Pulse Transit Time," Healthcare Innovation Conference (HIC), 2014, pp. 153-156.

13. M. Amin, Y. Heravi, and M. A. Khalilzadeh, "Designing and Constructing an Optical System to measure Continuous and Cuffless Blood Pressure Using Two Pulse Signals," Iranian Journal of Medical Physics vol. 10, no. 4, pp. 215-223, 2014.

14. M. Silva Vieira, T. Hussain, and C. Alberto Figueroa, "Patient-Specific Image-Based Computational Modeling in Congenital Heart Disease: A Clinician Perspective," J. Cardiol. Ther., vol. 2, no. 6, pp. 436-448, 2015.

15. N. Westerhof, J. W. Lankhaar, and B. E. Westerhof, "The arterial windkessel," Med. Biol. Eng. Comput., vol. 47, no. 2, pp. 131-141, 2009.

16. J.-Q. Engle, K. M.; Mei, T-S.; Wasa, M.; Yu, "Aging,Arterial Stiffness and Hypertension," Acc. Chem. Res., vol. 45, no. 6, pp. 788-802, 2008.

17. E. Pinto, "Blood pressure and ageing," Postgrad. Med. J., vol. 83, no. 976, pp. 109-114, 2007.

18. J. Fiala et al., "An implantable optical blood pressure sensor based on pulse transit time," Biomed. Microdevices, vol. 15, no. 1, pp. 73-81, 2013.

19. T. Wibmer et al., "Pulse transit time and blood pressure during cardiopulmonary exercise tests.," Physiol. Res., vol. 63, no. 3, pp. $287-$ 296,2014

20. K. Murakami and M. Yoshioka, "Pulse Transit Time Variability on a Range of Heart Rates between Resting and Elevated States," Int. Conf. Syst. Man, Cybern. 2015, pp. 1579-1582.

21. R. Wang, Wenyan Jia, Zhi-Hong Mao, R. J. Sclabassi, and Mingui Sun, "Cuff-free Blood Pressure Estimation using Pulse Transit Time and Heart Rate," 12th Int. Conf. Signal Process., 2014, pp. 115-118. 